\title{
Incidência e distribuição de Amblyomma cajennense em regiões corporais de eqüinos das raças Mangalarga Marchador e Bretão Postier, naturalmente infestados
}

\author{
MELISSA H. PINNA*, ARGEMIRO SANAVRIA**, \\ VITOR CÉZAR MACHADO*** y MARÍA C. de MORAIS****
}

\begin{abstract}
Amblyomma cajennense: ADULTS DISTRIBUTION AND INCIDENCE ON THE BODY OF NATURALLY INFESTED MANGALARGA MARCHADOR AND BRETÃO POSTIER HORSES
\end{abstract}

The objective of this study is to verify the incidence and the distribution of the tick Amblyomma cajennense tick in some infected regions of equines'body. Fifteen animals, 8 mares and 7 colts from Mangalarga Marchador and Bretão Postier races, raised in the Zootechny Institute Farm, Federal Rural University of Rio de Janeiro, were selected. From July, 1996 to May, 1997 (every 21 days) the animals were submitted to examination, mapping and ingurgitated females countig in 14 previously determined regions: face, lower jaw, neck, breast, shoulder-blade, armpits, former limbs, womb, back, flank, hip, udder, posterior limbs and perianal. The observed regions were classified in: anterior, posterior, median, right side and left side. The deviation analysis was made taking into account a random blocks delineation, whose scores were previously transformed on square root in order to decrease their dispersion, and subsequently analyzed by deviation analysis. During the observation time, the total of ticks was 1.434: 55.2\% corresponding to the anterior region and 44\% to the posterior region. It was also noticed a significant difference - 5\% - in June and April, occuring the higher parasitic incidence, regarding to September, with a lower incidence. On the right and left sides and median region of the animals body, the variation was: $27.7 \%, 30.1 \%$ and $42.2 \%$, respectively. The counting was higher in June; however, from August to September have showed lower incidences. On mares the distribution was: $59.5 \%$ on the anterior region and $40.5 \%$ on the posterior region: $32.9 \%$ on the right side, $28.1 \%$ on the left side and $39 \%$ on the median region. It was noticed significative difference among the countings averages on the three last regions in June (with higher incidence) and averages December, May, September, January and February averages (with lower incidences, respectively).

Key words: Amblyomma cajennense, ticks, horses.

\footnotetext{
* Médica Veterinária / Mestranda do Curso de Pós Graduação em Medicina Veterinária/UFRRJ hanzenpinna@bol.com.br

** Professor Adjunto IV / Instituto de Medicina Veterinária/UFRRJ - sanavria@ufrrj.br

*** Acadêmico do Curso de Medicina Veterinária da Universidade Estácio de Sá. **** Médica Veterinária / Mestranda do Curso de Pós Graduação em Medicina Veterinária UFRRJ
} 


\section{INTRODUÇÃO}

Amblyomma cajennense conhecido como "carrapato estrela", é um carrapato de regiões de clima tropical, que parasita preferencialmente eqüinos.

Estudos sobre a variação sazonal do $A$. cajennense, no noroeste da Argentina ${ }^{1}$, relatam que nas estações secas (outono e inverno) prevalecem ninfas e larvas, e adultos no verão. No Brasil2,3, estudos demonstraram que $A$. cajennense ocorre o ano todo nas pastagens do município de Itaguaí, Rio de Janeiro, mas há picos distintos de predominância de larvas, ninfas e adultos.

$\mathrm{O}$ uso indiscriminado de parasiticidas, por parte dos produtores e técnicos, pode levar a problemas referentes a intoxicações no homem e nos animais, devendo ser levado em consideração as recomendações e instruções dos fabricante ${ }^{4}$, Além disso, o uso incorreto predispõe ao aparecimento de resistência.

A resistência aos carrapaticidas tende a aumentar sua amplitude de distribuição pela propagação de cepas originalmente resistentes e/ ou pela contínua utilização de agentes químicos de controle; esse aumento, porém, não tem sido uniforme nem absoluto 5 .

A avaliação do comportamento dos referidos carrapatos no corpo dos hospedeiros é de imprescindível importância para o estabelecimento de métodos de controle viáveis e eficazes, e tem o objetivo de reduzir os prejuízos relacionados à equinocultura. $\mathrm{O}$ carrapato $A$. cajennense concentra-se preferencialmente, segundo $^{6}$, nos membros posteriores, axilas e região perianal.

O presente trabalho objetivou estudar a incidência e distribuição corpórea de carrapatos A. cajennense, em eqüinos das raças Mangalarga Marchador e Bretão Postier, naturalmente infestados, criados na Fazenda do Instituto de Zootecnia da Universidade Federal Rural do Rio de Janeiro (UFRRJ).

\section{MATERIAL E MÉTODOS}

O estudo foi desenvolvido no setor de Eqüinos do UFRRJ, situada a $22^{\circ} 55,08$, de latitude sul e $43^{\circ} 46$ ' 31 " de longitude oeste de Greenwich, com $13 \mathrm{~m}$ de altitude e $523 \mathrm{Km}^{2}$ de área.

Foram utilizados no experimento 180 eqüinos, registrados, das raças Mangalarga Marchador e
Bretão Postier, com idade variando de 6 meses a 20 anos, sendo mantidos em regime de pasto com exposição natural por Amblyomma cajennense, recebendo tratamento antiparasitário à base de piretróide.

No período de junho de 1996 a maio de 1997 , selecionou-se aleatoriamente 15 animais, sendo 8 éguas e 7 potras, os foram inspecionados individualmente a cada 21 dias, sendo feito contagem e mapeamento de teleógenas de 4.0 a $8.0 \mathrm{~mm}$, de acordo com sua localização em regiões corporais previamente estabelecidas: face, ganacha, pescoço, peito, paleta, axilas, membros anteriores, ventre, costado, flanco, anca, úbere, membros posteriores e perianal (Figuras 1 e 2).

Todos os dados meteorológicos utilizados na pesquisa foram levantados junto à Unidade da Estação Experimental de Itaguaí/PESAGRO-RJ, a fim de determinar a correlação entre a infestação por A.cajennense e os fatores climáticos da região (temperatura, umidade relativa do ar e precipitação pluvial).

Os resultados do índice de parasitismo nas diferentes regiões do corpo de equinos foram submetidos a análise de variância e foi estabelecida a correlação estatística entre as variáveis climatológicas e a incidência dos carrapatos. Para efeito de análise estatística, as regiões de observação foram agrupadas em: anterior, posterior, mediana, lado direito e esquerdo. A análise de variância foi feita considerando um delineamento com blocos ao acaso, onde os resultados foram previamente transformados em raiz quadrada, para diminuir a variabilidade de dispersão.

\section{RESULTADOS E DISCUSSÃO}

Verificou-se que apesar de ter sido realizado tratamento carrapaticida à base de piretróide, as tendências de maior ocorrência de $A$. cajennense não sofreram interferência. Este fato pode ser explicado, em parte, segundo ${ }^{7}$, como consequiência do curto período parasitário dos adultos e o longo intervalo entre banhos.

Constatou-se que a distribuição de adultos tende a atingir o ponto mais alto nos meses de Abril, Junho e Outubro, em ambas as categorias. Essa observação confirma os resultados publicados por investigadores ${ }^{2}$ referente a variação anual dos estádios de vida livre de $A$. cajennense onde foi constatado aumento da 


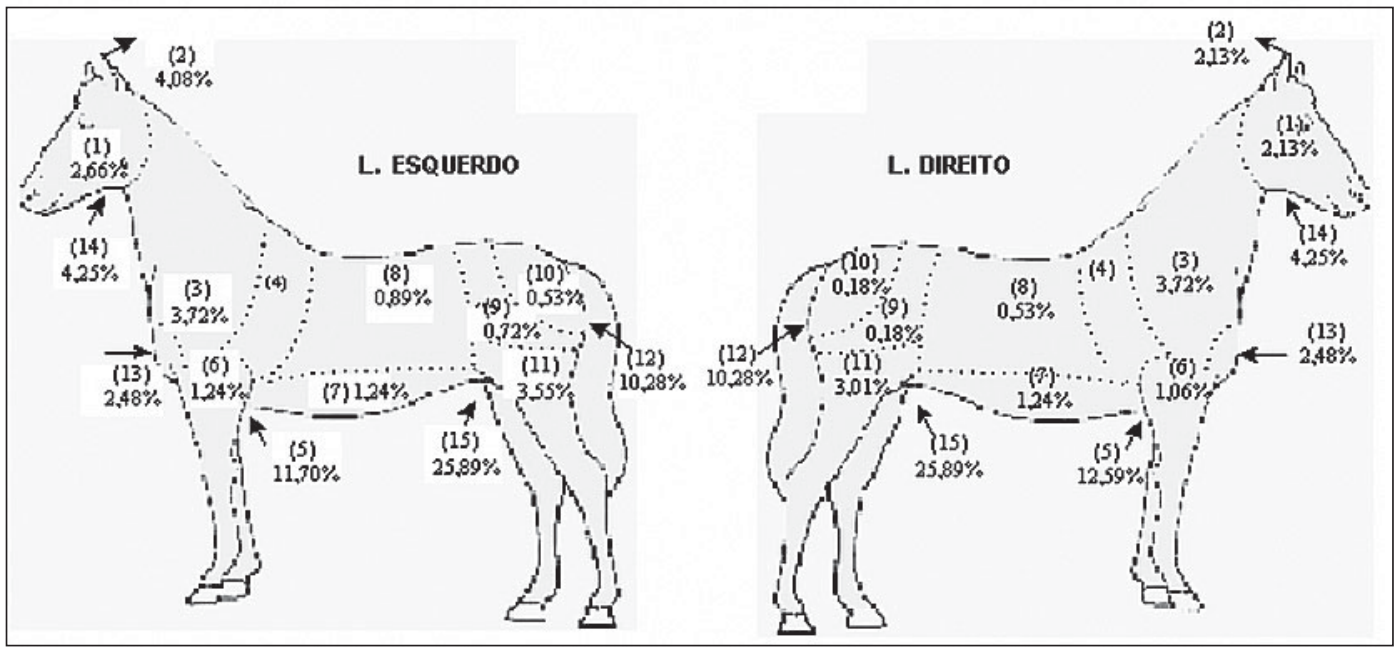

Figura 1. Incidência de Amblyomma cajennense em diferentes áreas corporais de potros. Face (1), Orelha (2), Pescoço (3), Paleta (4), Axilas (5), M. Anteriores (6), Ventre (7), Costado (8), Flanco (9), Anca (10), Membros Posteriores (11), Períneo (12), Peito (13), Ganacha (14) e Ubere (15).

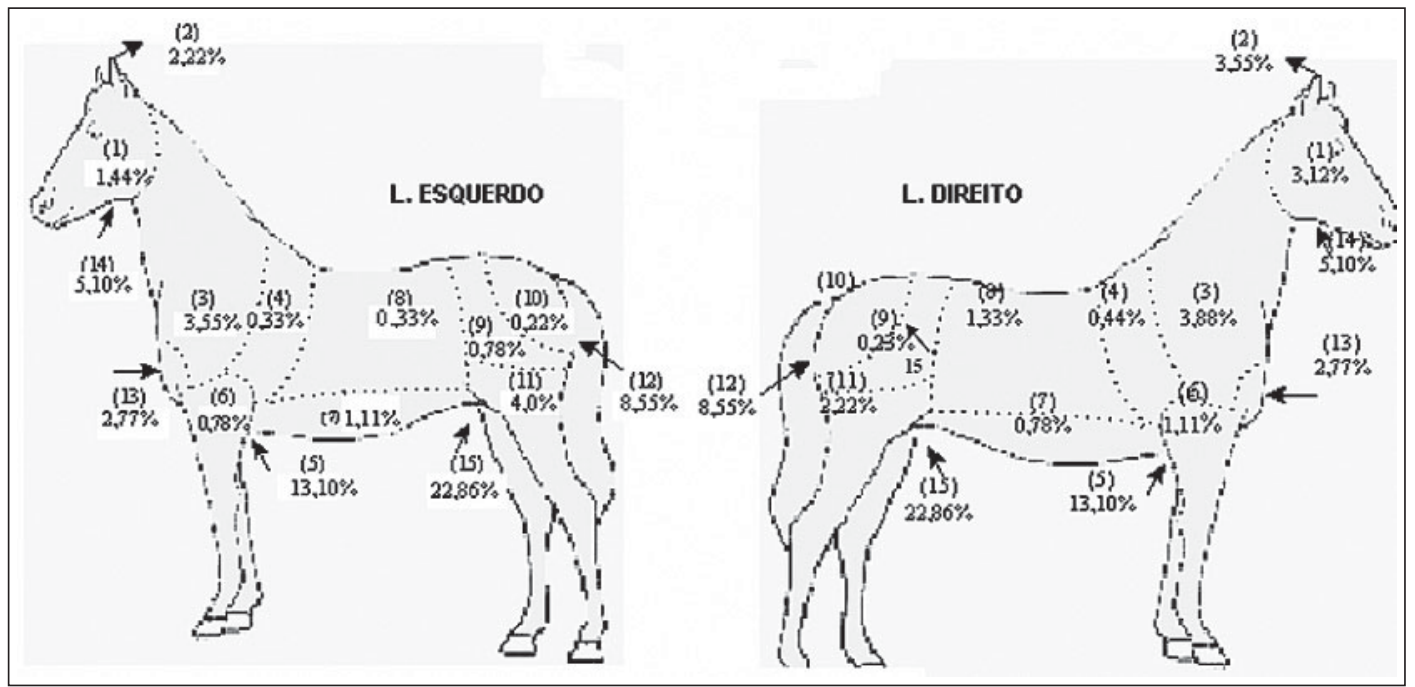

Figura 2. Incidência de Amblyomma cajennense em diferentes áreas corporais de éguas. Face (1), Orelha (2), Pescoço (3), Paleta (4), Axilas (5), M. Anteriores (6), Ventre (7), Costado (8), Flanco (9), Anca (10), Membros Posteriores (11), Períneo (12), Peito (13), Ganacha (14) e Ubere (15).

incidência de adultos na pastagem, durante o período de Outubro a Dezembro.

Os resultados acima coincidem com a disponibilidade de adultos nas pastagens submetidas ao pastoreio contínuo, descrito por outros autores ${ }^{8}$.

Constatou-se picos de incidência nos meses de Junho, Abril e Outubro, em ambas as categorias, respectivamente; sendo ainda verificado, uma correlação estatística ao nível de significância de $5 \%$ entre éguas e potros (Figura 3).

Para potros, o número de carrapatos mantevese constante nos meses de Novembro à Janeiro, época em que a precipitação pluviométrica foi, em média, igual ou superior a $200 \mathrm{~mm}$. Para éguas, foi observado que, no mesmo período, a variação tendeu a diminuir, porém, não ocorreu 


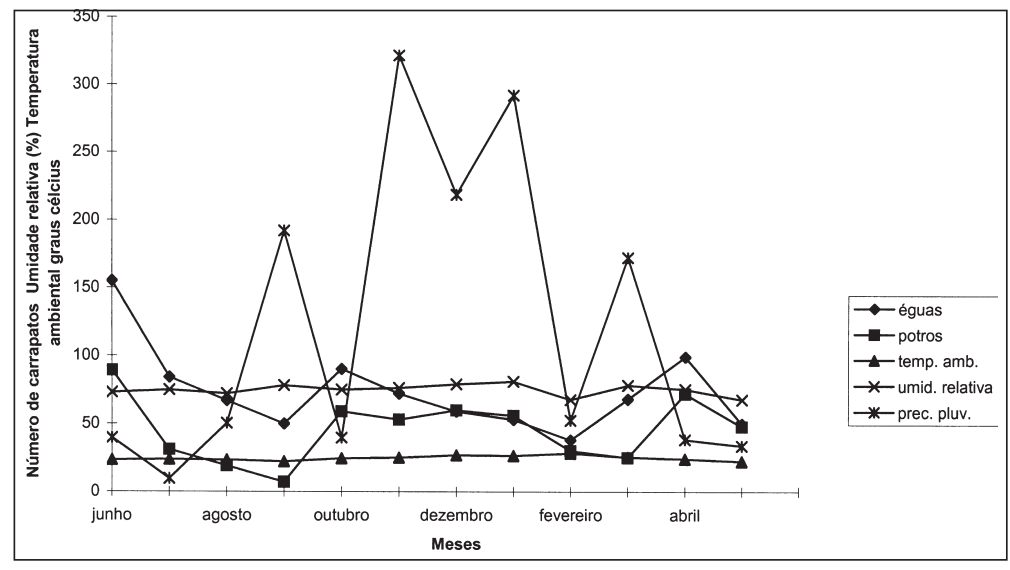

Figura 3. Relaçao entre os fatores climáticos e o nível de infestaçao mensal de éguas e potros. diferença significativa, comparativamente aos potros. Verificou-se que para os dois grupos a incidência parasitária teve uma tendência a aumentar no período de Fevereiro a Abril, época em que houve uma diminuição na precipitação pluviométrica. Esses resultados assemelham-se aos encontrados por $^{7}$, que indicam maiores infestações no período de Outubro a Maio.

Com relação à distribuição preferencial de $A$. cajennense no corpo dos eqüinos, naturalmente infestados, nas figuras 1 e 2 respectivamente, são demonstrados os percentuais de fixação, por região corporal, em éguas e potros. Houve diferença significativa quanto a fixação de carrapatos de acordo com a região do corpo do hospedeiro (ANOVA, p < 0,01).

Verificou-se que, para éguas, a maior concentração de carrapatos ocorreu nas axilas, úbere, pescoço, períneo, ganacha, face e membros posteriores. Por outro lado,as regiões do corpo que apresentaram menor frequência de parasitismo, foram: peito, membros anteriores, ventre, costado, flanco, paleta e anca.

Para potros, a maior concentração de carrapatos foi verificada na axila, seguida de úbere, períneo e membros posteriores. Por outro lado as regiões do corpo que apresentaram menor frequência de parasitismo, foram: membros anteriores e peito, costado, flanco e anca.

Segundo PRATA et $\mathrm{al}^{6}$ a maior concentração de carrapatos foi verificada nos membros posteriores $(36,60 \%)$, seguida de axilas $(15,90 \%)$, períneo $(13,70 \%)$, ventre $(10,61 \%)$ e face $(7,45 \%)$. Por outro lado, as regiões do corpo de menor frequência de parasitismo, foram: membros anteriores $(2,34 \%)$, paleta $(0,44 \%)$, costado $(0,28 \%)$, flanco $(0,11 \%)$ e anca $(0,02 \%)$. Para efeito de análise estatística, as regiões de observação foram agrupadas em: anterior, posterior, mediana, lado direito e esquerdo. Durante o período de observação obtivemos média de 11,20 carrapatos/potro e 13,83 carrapatos/égua.

Do total de 1.434 teleóginas coletadas em potros, 55,2\% correspondiam à região anterior e $44,8 \%$ à região posterior. Observou-se ainda a existência de diferença significativa (a nível de 5\%) nos meses de Junho e Abril, época de maior incidência parasitária, em relação ao mês de Setembro, onde houve menor incidência. Nos antímeros direito, esquerdo e região mediana do corpo dos animais, a oscilação foi de: $27,7,30,1$ e 42,2\%, respectivamente. A maior incidência foi constatada no mês de Junho, diferindo significativamente dos meses de Agosto e Setembro.

Nas éguas, a distribuição foi de: $59,5 \%$ na região anterior e $40,5 \%$ na região posterior; antímero direito $32,9 \%$, antímero esquerdo $28,1 \%$ e $39 \%$ na região mediana, observando-se diferença significativa entre as médias das contagens das três últimas regiões citadas entre o mês de Junho, com maior incidência e os meses de Dezembro, Maio, Setembro, Janeiro e Fevereiro, com menores incidências, respec-tivamente.

\section{RESUMO}

Com o objetivo de estudar a prevalência e a distribuição do carrapato Amblyomma cajennense nas diferentes regiões do corpo de eqüinos naturalmente infestados, foram selecionados 15 
animais, sendo oito éguas e sete potros, pertencentes às raças Mangalarga Marchador e Bretão Postier com idades variando entre 6 meses e 20 anos, criados na Fazenda do Instituto de Zootecnia da UFRRJ. No período de Junho de 1996 a Maio de 1997 (a cada 21 dias), os eqüinos, selecionados aleatoriamente, foram submetidos à inspeção visual, mapeamento e contagem de fêmeas ingurgitadas, em 14 regiões previamente estabelecidas: face, ganacha, pescoço, peito, paleta, axilas, membros anteriores, ventre, costado, flanco, anca, úbere, membros posteriores e perianal. Para a análise estatística, as regiões de observação foram agrupadas em: anterior, posterior, mediana, lado direito e lado esquerdo. A análise de variância foi feita considerando um delineamento com blocos ao acaso, onde as contagens foram previamente transformadas em raiz quadrada, para diminuir sua e posteriormente analisadas pela análise de variância. Durante o período de observação, o total de teleóginas foi de 1.434; sendo que, para potros, $55,2 \%$ correspondeu à região anterior e $44,8 \%$, à região posterior. Observou-se ainda a ocorrência de diferença significativa à nível de $5 \%$ nos meses de Junho e Abril, época de maior incidência parasitária, em relação ao mês de Setembro, onde houve menor incidência. Nos lados direito, esquerdo e região mediana do corpo dos animais, a oscilação foi de: 27,$7 ; 30,1$ e $42,2 \%$, respectivamente; bem como a contagem foi maior no mês de Junho e diferiu significativamente dos meses de Agosto e Setembro, que apresentaram menores incidências. Nas éguas, a distribuição foi de: $59,5 \%$ na região anterior e $40,5 \%$ na região posterior; $32,9 \%$ no lado direito, $28,1 \%$ no lado esquerdo e $39,0 \%$ na região mediana, observando-se diferença significativa entre as médias das contagens das três últimas regiões citadas, entre o mês de Junho (com maior incidência) e as dos meses de Dezembro, Maio, Setembro, Janeiro e Fevereiro (com menores ocorrências, respectivamente).

\section{REFERÊNCIAS}

1.- GUGLIELMONE AA, HADANI A. Amblyomma ticks found on cattle in the Northwest of Argentina. Ann Parasit 1982; 57: 91-7.

2.- SERRA-FREIRE N M. Epidemiologia de Amblyomma cajennense: ocorrência estacional e comportamento dos estádios não parasitários em pastagens no estado do Rio de Janeiro. Arq Univ Fed Rural Rio de J1982; 5: 111-4.

3.- SERRA-FREIRE N M, BONILHA P C, CAIAFFA R M et al. Avaliação da disponibilidade de estádios não parasitários de ixodídeos em pastagens submetidas ao pastejo contínuo por bovinos. Arq Univ Fed Rural Rio de J 1990; 13: 37-43.

4.- JORDÃO L P. Técnica elimina carrapato. Prod Leite 1981; 66: 26-30.

5.- BARRENTA S F. Resistência de las garrapatas a los ixodicidas. In Lucha contra las garrapatas del ganado. FAO. Est Agrop 1961; 54: 94-103.

6.- PRATA M C A, SANAVRIA A, SOUZA J R. Amblyomma cajennense (Fabricius, 1787) (Acari: Ixodidae): distribuition of adults on the body of naturaly infested horses. Rev Univ Rural Sér Ciênc Vida 1997 (no prelo).

7.- $\quad$ SOUZA A P, SERRA-FREIRE N M. Variação sazonal dos estádios adultos de Amblyomma cajennense e Anocentor nitens, como parasitas de cavalos, no município de Itaguaí, RJ, Brasil. Rev Brasil Parasitol Vet 1992; 1: 31-4.

8.- SOUZA A P, SERRA-FREIRE N M. Variação sazonal da fase não parasitária de Amblyomma cajennense e Anocentor nitens, no município de Itaguaí, RJ: avaliação epidemiológica e metodológica. Rev Univers Rural Sér Ciênc Vida 1994; 16: 67-74. 